\title{
ECG-Gated Interventional Cardiac Reconstruction for Non-periodic Motion
}

\author{
Christopher Rohkohl ${ }^{1,2}$, Günter Lauritsch ${ }^{2}$, \\ Lisa Biller ${ }^{2}$, and Joachim Hornegger ${ }^{1}$ \\ 1 Pattern Recognition Lab, Department of Computer Science, \\ Friedrich-Alexander University Erlangen-Nuremberg, \\ 91058 Erlangen, Germany \\ 2 Siemens AG, Healthcare Sector, 91301 Forchheim, Germany
}

\begin{abstract}
The 3-D reconstruction of cardiac vasculature using $\mathrm{C}$-arm $\mathrm{CT}$ is an active and challenging field of research. In interventional environments patients often do have arrhythmic heart signals or cannot hold breath during the complete data acquisition. This important group of patients cannot be reconstructed with current approaches that do strongly depend on a high degree of cardiac motion periodicity for working properly. In a last year's MICCAI contribution a first algorithm was presented that is able to estimate non-periodic 4-D motion patterns. However, to some degree that algorithm still depends on periodicity, as it requires a prior image which is obtained using a simple ECG-gated reconstruction. In this work we aim to provide a solution to this problem by developing a motion compensated ECG-gating algorithm. It is built upon a 4-D timecontinuous affine motion model which is capable of compactly describing highly non-periodic motion patterns. A stochastic optimization scheme is derived which minimizes the error between the measured projection data and the forward projection of the motion compensated reconstruction. For evaluation, the algorithm is applied to 5 datasets of the left coronary arteries of patients that have ignored the breath hold command and/or had arrhythmic heart signals during the data acquisition. By applying the developed algorithm the average visibility of the vessel segments could be increased by $27 \%$. The results show that the proposed algorithm provides excellent reconstruction quality in cases where classical approaches fail. The algorithm is highly parallelizable and a clinically feasible runtime of under 4 minutes is achieved using modern graphics card hardware.
\end{abstract}

\section{Introduction}

\subsection{Purpose of This Work}

One key component of image guidance in the field of interventional cardiology is three-dimensional image information before, during and after interventional procedures. Three-dimensional image data can support complex interventional procedures, such as transcutaneous valve replacement, interventional therapy of

T. Jiang et al. (Eds.): MICCAI 2010, Part I, LNCS 6361, pp. 151-158, 2010.

(C) Springer-Verlag Berlin Heidelberg 2010 
atrial fibrillation, implantation of biventricular pacemakers and the assessment of myocardial perfusion.

With the technology of C-arm CT it is possible to reconstruct intraprocedural 3-D images from angiographic projection data [1. Currently, the major limitation of this technology is its insufficient temporal resolution which limits the visualization of fast moving parts of the heart. Due to the long acquisition time of several seconds, at which a couple of heart beats and breathing motion can occur, motion related image artifacts, e.g. blurring or streaks are observed. Therefore it is essential to develop algorithms that can cope with both cardiac and respiratory motion.

\subsection{State-of-the-Art}

Based on the electrocardiogram (ECG) a relative cardiac phase is assigned to each projection image assuming a periodic heart motion [2. The phase information is used for a phase-correlated reconstruction by gating or motion estimation and compensation. A gated reconstruction takes only those images into account that lie inside a defined phase window, that is centered at the targeting cardiac phase to be reconstructed [2, 3. This is, however, not ideal in terms of missing data for single-run acquisitions. The incomplete data leads to streak artifacts and a poor signal-to-noise ratio. To increase the data usage, the cardiac motion is estimated and motion compensated reconstruction algorithms are applied [4, 2, 5, 6, 7. Reasonable results are provided in the presence of regular heart rates without breathing or other patient motion. However, in the field of intraprocedural cardiac reconstruction, the patients suffer from heart diseases and cannot completely hold breath, stay still or have irregular heart beats. Those aspects do conflict with the periodicity assumption of ECG-based methods.

In literature these problems were addressed by approximate 2-D corrections in the projection image [4,8]. In a last year's MICCAI contribution [9], a first algorithm was presented that is able to estimate true 4-D non-periodic motion patterns using a time-continuous B-spline motion model (NOPMEC). However, this algorithms requires an initial 3-D reference image as a priori information. This reference is easy to obtain for almost periodic motion by ECG-gated reconstruction. However, strong respiratory motion and severe arrhythmia can make it impossible to obtain a sufficient initial image. This weak point is to be solved in this paper.

\section{Affine 4-D Motion Estimation and Reconstruction}

\subsection{General Idea}

In previous investigations, e.g. by Shechter et al. [10] it has been shown that heart phase variations and respiratory motion of cardiac vasculature can be modelled using global transformations, i.e. rigid body transformations or affine transformations. In the following sections an algorithm for the estimation of such an affine motion between the different heart beats is presented. 


\subsection{Affine 4-D Time-Continuous Motion Model}

We assume a time-continuous motion model function that maps a voxel $\boldsymbol{x}=$ $\left(x_{0}, x_{1}, x_{2}\right)^{T}$ to a new voxel location $\boldsymbol{x}^{\prime}$ for each time when a projection image is acquired. It is conceptionalized by a function $M: \mathbb{N} \times \mathbb{R}^{3} \times \mathbb{S} \mapsto \mathbb{R}^{3}$ with $M(i, \boldsymbol{x}, \boldsymbol{s})=\boldsymbol{x}^{\prime}$ transforming the voxel coordinate $\boldsymbol{x}$ at the time of the $i$-th projection image. The mapping is based on the motion model paramters $s \in \mathbb{S}$. In this work a global affine motion model is used which is defined in the following.

A set of time points is created and each point in time is assigned 12 affine parameters that describe the affine transformation at that moment. The set of time points is determined from the ECG-signal. We are choosing two time points per heart beat closest to the reference heart phase $h_{\mathrm{r}}$ and to an additional phase $h_{\mathrm{r}}+\Delta h$. For a heart phase $h$ this set of projection images is denoted $\mathcal{N}_{h}$. The complete set $L$ of temporal control points is then given by

$$
L=\{1, N\} \cup \mathcal{N}_{h_{\mathrm{r}}} \cup \mathcal{N}_{h_{\mathrm{r}}+\Delta h},
$$

where the number of projections ranges from 1 to $N$. The first and last projection image is added such that no boundary problems will occurr in the following.

The affine parameters $s_{l} \in \mathbb{R}^{12}$ for a single time point $l \in L$ are collected in a vector $\boldsymbol{s}_{l}=\left(t_{0}, t_{1}, t_{2}, \alpha_{0}, \alpha_{1}, \alpha_{2}, a_{0}, a_{1}, a_{2}, b_{0}, b_{1}, b_{2}\right)^{T}$, where $t_{i}$ represents the translation along, $\alpha_{i}$ the rotation around, $a_{i}$ the scaling along and $b_{i}$ the shearing of the $i$-th coordinate axis. The complete parameter vector $s \in \mathbb{S}, \mathbb{S}=\mathbb{R}^{12|L|}$ is then given by

$$
s=\left(s_{L_{1}}, \ldots, s_{L_{|L|}}\right)^{T}
$$

with $L_{i}$ being the $i$-th smallest element of $L$. For an arbitrary projection image $i$ the affine transform parameters $\tilde{\boldsymbol{s}}_{i}$ are then obtained by temporal interpolation of each component. In our work a cubic B-spline interpolation [1] has been used. The final motion model is then formally given by

$$
M(i, \boldsymbol{x}, s)=\boldsymbol{x}^{\prime} \quad \text { with } \quad \boldsymbol{A}_{\tilde{s_{i}}}\left(\begin{array}{c}
\boldsymbol{x} \\
1
\end{array}\right) \cong\left(\begin{array}{c}
\boldsymbol{x}^{\prime} \\
1
\end{array}\right)
$$

where $\boldsymbol{A}_{\tilde{s_{i}}}$ is the affine transformation matrix in homogenous coordinates for the affine parameters $\tilde{\boldsymbol{s}}_{i}$. The formation of the affine transformation matrix is straightforward and can be found e.g. in [12].

\subsection{Motion Compensated ECG-Gated Reconstruction}

Projection Image Preprocessing. For motion estimation we are only interested in the motion of the cardiac vasculature. Therefore we apply a background reduction technique previously proposed by Hansis et al. 3] using a morphological top-hat filter. In the following this preprocessed projection data will be referred to by the function $p: \mathbb{N} \times \mathbb{R}^{2} \mapsto \mathbb{R}$ where $p(i, \boldsymbol{u})$ returns the value of the $i$-th preprocessed projection image at the pixel $\boldsymbol{u}$. 
Reconstruction Algorithm. For motion estimation and correction a dynamic reconstruction $f(\boldsymbol{x}, \boldsymbol{s})$ is defined. The function $f$ returns the reconstructed object value at a voxel $\boldsymbol{x}$ based on the motion model parameters $\boldsymbol{s}$. In this paper, an ECG-gated motion compensated FDK reconstruction method is utilized [2,5]. The ECG-gating is performed by applying a weighting factor $\lambda$ to each image which is calculated from the distance to the reference heart phase. The dynamic ECG-gated FDK reconstruction $f_{h_{\mathrm{r}}}: \mathbb{R}^{3} \times \mathbb{S} \mapsto \mathbb{R}$ is then given by

$$
f_{h_{\mathrm{r}}}(\boldsymbol{x}, \boldsymbol{s})=\sum_{i} \lambda\left(i, h_{\mathrm{r}}\right) \cdot w(i, M(i, \boldsymbol{x}, \boldsymbol{s})) \cdot p_{F}(i, A(i, M(i, \boldsymbol{x}, \boldsymbol{s}))) .
$$

The function $w: \mathbb{N} \times \mathbb{R}^{3} \mapsto \mathbb{R}$ is the distance weight of the FDK-formula. The pre-processed, filtered and redundancy weighted projection data is accessed by the function $p_{F}: \mathbb{N} \times \mathbb{R}^{2} \mapsto \mathbb{R}$ where $p_{F}(i, \boldsymbol{u})$ returns the value of the $i$-th image at the pixel $\boldsymbol{u}$. The pixel location $\boldsymbol{u}$ is determined by the perspective projection $A: \mathbb{N} \times \mathbb{R}^{3} \mapsto \mathbb{R}^{2}$, where $A(i, \boldsymbol{x})=\boldsymbol{u}$ maps a voxel $\boldsymbol{x}$ to a pixel location $\boldsymbol{u}$ in the $i$-th projection image. The function $\lambda$ is a weighting function adapted from [5] that is used for obtaining an ECG-phase correlated reconstruction for the heart phase $h_{\mathrm{r}} \in[0,1]$. It is given by

$$
\lambda\left(i, h_{\mathrm{r}}\right)= \begin{cases}\cos ^{\beta}\left(\frac{d\left(h(i), h_{\mathrm{r}}\right)}{\omega} \pi\right) & \text { if } d\left(h(i), h_{\mathrm{r}}\right) \leq \frac{\omega}{2} \\ 0 & \text { otherwise }\end{cases}
$$

where $h(i)$ is the heart phase of the $i$-th projection image and $\omega \in(0,1]$ is the width of the non-zero support region of the weighting function. The parameter $\beta \in[0, \infty)$ controls the shape of the support region. The distance function $d\left(h_{1}, h_{2}\right)=\min _{c \in\{0,1,-1\}}\left|h_{1}-h_{2}+c\right|$ returns the distance between two relative heart phases.

\subsection{Objective Function for Motion Estimation}

Motion estimation is formulated as a multi-dimensional optimization problem where the motion model parameters $\hat{s} \in \mathbb{S}$ maximizing the objective function $\mathcal{L}: \mathbb{S} \mapsto \mathbb{R}$ need to be estimated. The objective function introduced in this paper is motivated by the basic relationship of the motion compensated reconstruction $f$ with the measured projection data $p$. Maximum intensity projections of the motion state observed in a projection image can be created from a motion compensated reconstruction $f(\boldsymbol{x}, s)$ by dynamic forward projection:

$$
r(i, \boldsymbol{u}, \boldsymbol{s})=\max _{x \in L_{i, u}} f_{h_{\mathrm{r}}}\left(M^{-1}(i, \boldsymbol{x}, \boldsymbol{s}), \boldsymbol{s}\right) .
$$

The function $r: \mathbb{N} \times \mathbb{R}^{2} \times \mathbb{S} \rightarrow \mathbb{R}$ returns the dynamic maximum intensity forward projection of the ECG-gated and motion compensated reconstruction $f_{h_{\mathrm{r}}}$. The voxels on the straight measurement ray $L_{i, u}$ of the $i$-th image hitting the detector at pixel $\boldsymbol{u}$ are transformed by the inverse motion model to consider the motion state observed at the projection image $i$. The matching of the measured 
and preprocessed data $p$ and the forward projected data $r$ is assessed by computing the average normalized cross-correlation (NCC). Formally, the objective function to be maximized is then given by:

$$
\mathcal{L}(\boldsymbol{s})=\arg \max _{\boldsymbol{s} \in \mathbb{S}} \frac{1}{\nu} \sum_{i}^{N}\left(\lambda\left(i, h_{\mathrm{r}}\right) \sum_{\boldsymbol{u}} \frac{\left(p(i, \boldsymbol{u})-\mu_{p_{i}}\right)\left(r(i, \boldsymbol{u}, \boldsymbol{s})-\mu_{r_{i, s}}\right)}{\sigma_{p_{i}} \sigma_{r_{i, s}}}\right)
$$

with the normalizing factor $\nu=\left(I_{\mathrm{p}}-1\right) \sum_{i}^{N} \lambda\left(i, h_{\mathrm{r}}\right), I_{\mathrm{p}}$ being the number of image pixels $\boldsymbol{u}$ of a projection and $\mu, \sigma$ being the mean and standard deviation of the subscripted image. The value of the objective function ranges $\mathcal{L}(s) \in$ $[-1,1]$ with the maximum value representing a perfect linear relationship of the measured and forward projected data. The NCC of the $i$-th projection image pair is weighted by the gating function $\lambda$ as it characterizes the influence on the dynamic ECG-gated reconstruction.

\subsection{Optimization Strategy}

For maximization of Eq. (7) a stochastic gradient ascent method is used. In each iteration one of the temporal points is selected with a certain probability. For initialization, all time points are assigned the same probability. The gradient is computed using finite differences by varying the 12 affine parameters for the selected time point. Next, one step is taken into the gradient direction with a fixed step size. The probability of the time point of being selected in the next iteration, is set proportional to the increase of cost function value. Optimization stops after a certain number of iterations or if the convergence ratio drops below a certain threshold. This kind of stochastic selection of parameters for the gradientbased optimization procedure has the benefit that it picks out the most beneficial parameters. In that way a fast convergence with as few as possible cost function evaluations was found.

\subsection{Implementation Details}

One evaluation of the objective function Eq. (7) comprises an ECG-gated reconstruction, an ECG-gated forward projection and the computation of the quality measure. Each step is very well parallelizable on the graphics card using CUDA [13]. The backprojection of the FDK-reconstruction and forward projection are based on projection matrices. The affine matrix $\boldsymbol{A}_{\tilde{s_{i}}}$ in Eq. (3) only depends on the projection geometry and is independent of the voxel location. This allows us to replace the voxel-wise computation of the motion transform $M$ by a right-side multiplication of the projection matrix with the affine transformation matrix. Consequently, no additional overhead is introduced during forward or backward projection operations.

\section{Experimental Setup}

The presented algorithm is integrated into the non-periodic motion estimation and compensation scheme of [9] (NOPMEC). It requires as input a prior 
image which is in the standard version a simple ECG-gated reconstruction. For our experiments the standard version is compared with a version that uses the proposed ECG-gating for non-periodic motion presented in this paper.

Datasets of left coronary arteries divided into two groups are reconstructed. The first group $G_{1}$ consists of five cases with good breath hold and a regular heart rate. The second group $G_{2}$ consists of five cases that have ignored the breath hold command and/or had arrhythmic heart signals during the data acquisition. For both groups the coronary segments $S_{5}$ to $S_{15}$, named according to the guidelines of the American Heart Association 14 are identified and the visibility is rated with a value from zero to three with the following meaning: 0 (not visible), 1 (partially visible, strong artifacts), 2 (completely visible, some artifacts), 3 (close to perfect).

The datasets were acquired on an Artis Zeego C-arm system (Siemens AG, Healthcare Sector, Forchheim, Germany). They consist each of $N=133$ projection images acquired in 5 seconds with a size of $960 \times 1240$ pixels at an isotropic resolution of $0.32 \mathrm{~mm} /$ pixel. The number of iterations was set to 300 . The reconstruction parameters were set to $h_{\mathrm{r}}=0.7, h_{\mathrm{r}}+\Delta h=0.2, \omega=0.2, \beta=0$. Image reconstruction is performed on an image volume of $20^{3} \mathrm{~cm}^{3}$ distributed on a $256^{3}$ voxel grid. The parameters of the NOPMEC algorithm 9] were set to 5 spatial and 35 temporal control points with 100 iterations.

\section{Results and Discussion}

The average rating for each of the segments $S_{5}$ to $S_{15}$ is listed separately for each patient group and for both algorithms in Table 1, The performance of the proposed method does not significantly increase the visibility for group $G_{1}$ $(1.3 \%)$. This is reasonable, as this group contains highly periodic data for which the simple ECG-gating already works well. The 3-D reconstruction of an example case from group $G_{1}$ is depicted in Fig. 1d,

Table 1. Results from the evaluation of the clinical data. For each segment the visibility for both patient groups is analyzed. Results are given for the standard algorithm and the proposed method.

\begin{tabular}{|c|c|c|c|c|c|c|}
\hline & standarc & $\begin{array}{l}\text { Group } G \\
\text { d propose }\end{array}$ & increase & standard & $\begin{array}{l}\text { Group } G \\
\text { d propose }\end{array}$ & increase \\
\hline$S_{5}$ & 3.00 & 3.00 & $0.0 \%$ & 3.00 & 3.00 & $0.0 \%$ \\
\hline$S_{6}$ & 2.80 & 2.80 & $0.0 \%$ & 2.60 & 2.80 & $7.7 \%$ \\
\hline$S_{7}$ & 2.80 & 2.80 & $0.0 \%$ & 2.00 & 2.20 & $10.0 \%$ \\
\hline$S_{8}$ & 2.80 & 2.80 & $0.0 \%$ & 2.40 & 2.80 & $16.7 \%$ \\
\hline$S_{9}$ & 2.60 & 2.80 & $7.7 \%$ & 2.00 & 2.60 & $30.0 \%$ \\
\hline$S_{10}$ & 2.40 & 2.40 & $0.0 \%$ & 1.00 & 1.75 & $75.0 \%$ \\
\hline$S_{11}$ & 3.00 & 3.00 & $0.0 \%$ & 2.20 & 2.60 & $18.2 \%$ \\
\hline$S_{12}$ & 2.80 & 2.80 & $0.0 \%$ & 0.75 & 1.50 & $100.0 \%$ \\
\hline$S_{13}$ & 2.60 & 2.60 & $0.0 \%$ & 1.80 & 2.60 & $44.4 \%$ \\
\hline$S_{14}$ & 2.20 & 2.20 & $0.0 \%$ & 1.00 & 1.50 & $50.0 \%$ \\
\hline$S_{15}$ & 2.80 & 3.00 & $7.1 \%$ & 1.75 & 2.75 & $57.1 \%$ \\
\hline Avg. & 2.71 & 2.75 & $1.3 \%$ & 1.86 & 2.37 & $27.3 \%$ \\
\hline
\end{tabular}




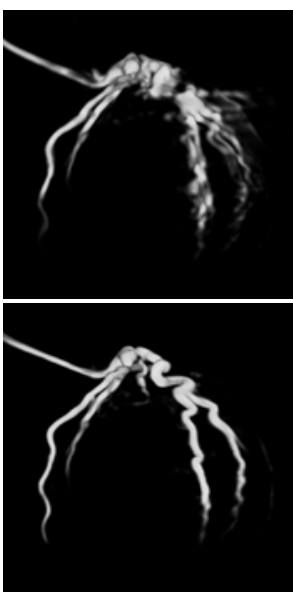

(a) Ex. case of $G_{2}$

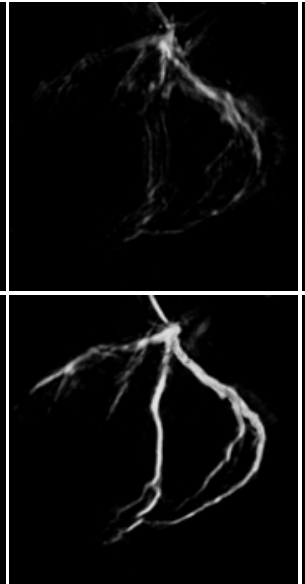

(b) Ex. case of $G_{2}$

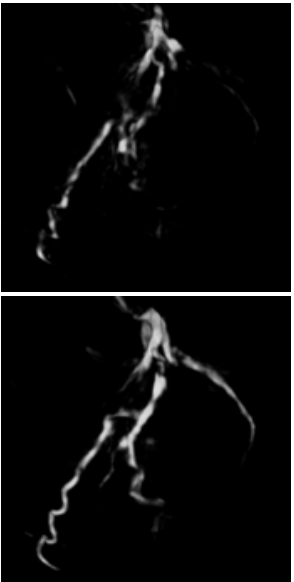

(c) Ex. case of $G_{2}$

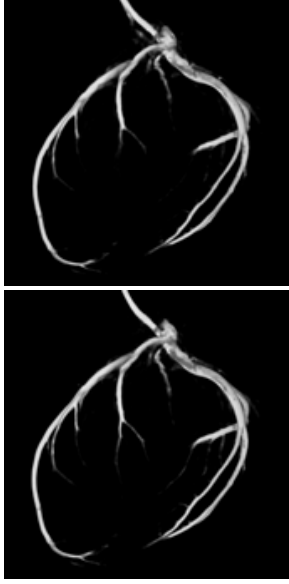

(d) Ex. case of $G_{1}$

Fig. 1. Example reconstruction results for the clinical datasets using the standard algorithm (top row) and the proposed approach (bottom row). The first three datasets belong to the patient group $G_{2}$ while the last one belongs to $G_{1}$.

For patient group $G_{2}$ the situation changes. The average visibility can be increased significantly $(27.3 \%)$ using the method proposed in this paper. Three of the cases are depicted in Fig. 1a to Fig. 1c to provide a visual impression of the quality increase. By applying the proposed method, the quality can be increased drastically (bottom row) in terms of intensity, connectedness, artifact level and smoothness of the vessels. In summary the proposed method never decreased the visibility of any of the vessel segments and provided superior image quality in cases of non-periodic motion (group $G_{2}$ ). The runtime of our algorithm was below 4 minutes in all cases.

\section{Conclusions and Outlook}

A major clinical challenge of $\mathrm{C}$-arm based cardiac vasculature reconstruction is non-periodic motion. In this paper a framework for the ECG-gated 3-D reconstruction with affine motion correction for non-periodic motions has been introduced. It has been integrated into the NOPMEC algorithm for obtaining a prior image. It is demonstrated that for non-periodic motion, using simple ECG-gating as prior image fails, while the visiblity of the vasculature could be increased in average by $27 \%$, using the motion compensated ECG-gating presented in this paper. In summary, with the proposed method an important step towards the clinical usability has been made by addressing patient groups which cannot be reconstructed with previous methods.

Disclaimer: The concepts and information presented in this paper are based on research and are not commercially available. 


\section{References}

1. Hetterich, H., Redel, T., Lauritsch, G., Rohkohl, C., Rieber, J.: New X-ray imaging modalities and their integration with intravascular imaging and interventions. In: The International Journal of Cardiovascular Imaging (2009) (online) (to be printed)

2. Schäfer, D., Borgert, J., Rasche, V., Grass, M.: Motion-compensated and gated cone beam filtered back-projection for 3-d rotational x-ray angiography. IEEE Transactions on Medical Imaging 25(7), 898-906 (2006)

3. Hansis, E., Schafer, D., Dossel, O., Grass, M.: Evaluation of iterative sparse object reconstruction from few projections for 3-d rotational coronary angiography. IEEE Transactions on Medical Imaging 27(11), 1548-1555 (2008)

4. Blondel, C., Malandain, G., Vaillant, R., Ayache, N.: Reconstruction of coronary arteries from a single rotational x-ray projection sequence. IEEE Transactions on Medical Imaging 25(5), 653-663 (2006)

5. Rohkohl, C., Lauritsch, G., Nöttling, A., Prümmer, M., Hornegger, J.: C-arm ct: Reconstruction of dynamic high contrast objects applied to the coronary sinus. In: IEEE NSS-MIC, Dresden, Germany, M10-M328 (October 2008)

6. Prümmer, M., Hornegger, J., Lauritsch, G., Wigström, L., Girard-Hughes, E., Fahrig, R.: Cardiac c-arm ct: A unified framework for motion estimation and dynamic ct. IEEE Trans. Med. Imaging 28(11), 1836-1849 (2009)

7. Keil, A., Vogel, J., Lauritsch, G., Navab, N.: Dynamic cone beam reconstruction using a new level set formulation. In: Yang, G.-Z., Hawkes, D., Rueckert, D., Noble, A., Taylor, C. (eds.) MICCAI 2009. LNCS, vol. 5762, pp. 389-397. Springer, Heidelberg (2009)

8. Hansis, E., Schäfer, D., Dössel, O., Grass, M.: Projection-based motion compensation for gated coronary artery reconstruction from rotational x-ray angiograms. Physics in Medicine and Biology 53(14), 3807-3820 (2008)

9. Rohkohl, C., Lauritsch, G., Prümmer, M., Hornegger, J.: Interventional 4-d motion estimation and reconstruction of cardiac vasculature without motion periodicity assumption. In: Yang, G.-Z., Hawkes, D., Rueckert, D., Noble, A., Taylor, C. (eds.) MICCAI 2009. LNCS, vol. 5761, pp. 132-139. Springer, Heidelberg (2009)

10. Shechter, G., Ozturk, C., Resar, J.R., McVeigh, E.R.: Respiratory motion of the heart from free breathing coronary angiograms. IEEE Transactions on Medical Imaging 23(8), 1046-1056 (2004)

11. Unser, M.: Splines: A perfect fit for signal and image processing. IEEE Signal Processing Magazine 16(6), 22-38 (1999); IEEE Signal Processing Society's 2000 magazine award

12. Hartley, R.I., Zisserman, A.: Multiple View Geometry in Computer Vision, 2nd edn. Cambridge University Press, Cambridge (2004) ISBN: 0521540518

13. Rohkohl, C., Keck, B., Hofmann, H., Hornegger, J.: RabbitCT - an open platform for benchmarking 3D cone-beam reconstruction algorithms. Medical Physics 36, 3940-3944 (2009)

14. Austen, W., Edwards, J., Frye, R., Gensini, G., Gott, V., Griffith, L., McGoon, D., Murphy, M., Roe, B.: A reporting system on patients evaluated for coronary artery disease. Circulation 51, 5-40 (1975) 